\title{
Los desafíos de la interculturalidad frente a la persistente homogeneización: una reflexión desde la realidad de Brasil y México
}

\author{
NASCIMENTO, Raimundo Nonato Ferreira do ${ }^{1}$ \\ LOBATOS, Violeta Denis Jiménez ${ }^{2}$
}

\section{RESUMEN}

La interculturalidad y la educación intercultural en Brasil y México, aún pueden ser vistos como campos emergentes. Las reflexiones sobre tales conceptos son una constante en los espacios académicos, en el área de planeación de políticas institucionales, mientras que en la intervención pedagógica se muestran como algo novedoso. En ese sentido, el modelo educativo intercultural que presenta el gobierno brasileño a las poblaciones indígenas y el gobierno mexicano a la población en general son políticas que intentan promover el reconocimiento y el respeto a las diferentes culturas, no obstante, en la práctica han encontrado grandes desafíos. Sobre este tema se discute en el presente artículo. Se reflexiona sobre los límites y los desafíos de la práctica de la educación intercultural en Brasil y en México, tomando como base el análisis de las normas legales propuestas por el Estado y relacionándolas con la realidad de las escuelas, para con ello mostrar algunos desafíos que se enfrentan en ambos países.

Interculturalidad. Educación intercultural. Brasil y México.

\section{Os desafios da interculturalidade diante da persistente homogeneização: uma reflexão a partir da realidade do Brasil e do México}

\section{RESUMO}

A interculturalidade e a educação intercultural, tanto no Brasil como no México, ainda podem ser vistas como um campo em emergência. Mesmo que as reflexões sobre tais conceitos sejam uma constante nos espaços acadêmicos, na área de planejamento de políticas institucionais ou mesmo como intervenção

\footnotetext{
${ }^{1}$ Doutor em Antropologia. Professor Adjunto II do Departamento de Ciências Sociais da Universidade Federal do Piauí. Professor Adjunto I, do Centro de Ciências Humanas e Letras, Departamento de Ciências Sociais da UFPI. Lider do GPADIE - Grupo de estudos e pesquisa em Antropologia, Diversidade, interculturalidade e educação. Email: nonatorr.33@gmail.com. ORCID: https://orcid.org/0000-0003-2667-7928.

${ }^{2}$ Doctora en Investigación Educativa. Profesora de História Secundária en la Secretaria de Educación de Vera Cruz, México. Educación Intercultural de Vera Cruz. Email: violetadjl@gmail.com. ORCID: https://orcid.org/0000-0003-2050-9026.
}

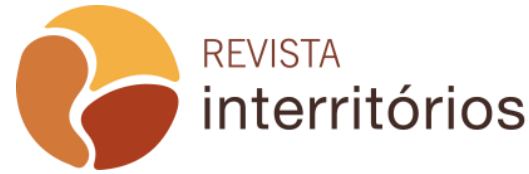


pedagógica se mostra como algo novo. Nesse sentido, o modelo educativo intercultural destinado as populações indígenas com o intuito de promover um reconhecimento, valorização e respeito as diferentes culturas, tem encontrado desafios para sua efetivação. É, portanto, sobre esse tema que aborda o presente trabalho. Nosso objeto, é refletir sobre os limites e desafios do fazer educativo intercultural no Brasil e no México. Por meio de uma análise comparativa das normas legais, ou seja, do que se propõe com a realidade pratica da escola que pretendemos demonstrar alguns desafios.

Interculturalidade. Educação intercultural. Brasil e México.

The challenges of interculturality in the face of a persistent homogenization: a reflection from the reality of Brazil and Mexico

\section{ABSTRACT}

Interculturality and intercultural education in both Brazil and Mexico can still be seen as an emerging field. Even though reflections on such concepts are a constant in academic spaces, in the area of institutional policy planning or even as a pedagogical intervention it is shown as something new. In this sense, the intercultural educational model intended for indigenous populations in order to promote recognition, appreciation and respect for different cultures has encountered challenges to become effective. Therefore, this theme is what this paper addresses. Our purpose is to reflect on the limits and challenges of intercultural educational work in Brazil and Mexico. By means of a comparative analysis of the legal norms, that is, what is proposed with the practical reality of schools, we intend to demonstrate some challenges.

Interculturality. Intercultural education. Brazil and Mexico.

\section{Le sfide dell'interculturalità di fronte all'omogeneizzazione persistente: un riflesso della realtà del Brasile e del Messico}

\section{RIASSUNTO}

un'interculturalità e un'educazione interculturale, sia non in Brasile che in Messico, possono essere viste come un campo di emergenza. Mesmo che mentre rifletti su questi consigli è uno spazio accademico costante, un'area di pianificazione delle politiche istituzionali o mesmo come l'intervento pedagogico viene mostrato come qualcosa di nuovo. In questo senso, o modello educativo interculturale inteso come popolazione indigena o intuito per promuovere il riconoscimento, la valorizzazione e il rispetto di culture diverse, ha trovato difficoltà nella sua efficacia. É, quindi, su questo tema che tratta o presenta lavori. II nostro scopo è quello di confutare i limiti e le sfide delle attività educative

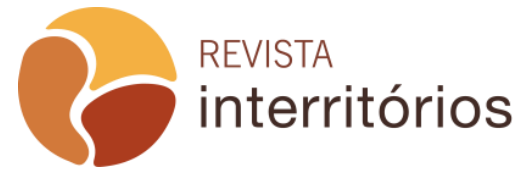


interculturali non in Brasile e non in Messico. Tramite un'analisi comparativa delle norme giuridiche, cioè come realtà pratica della scuola intendiamo dimostrare alcune sfide. "

Interculturalismo. Educazione Interculturale. Brasile e Messico.

\section{INTRODUCCIÓN}

En los últimos años, hemos visto que las temáticas relacionadas con la interculturalidad y la educación intercultural se han tornado un punto central en los debates académicos. En muchos de ellos, se han propuesto estrategias y soluciones a los desafíos que se imponen en la práctica de la educación intercultural. En este sentido, el presente trabajo, tiene como objetivo realizar una reflexión sobre los desafíos y los límites que se han puesto en marcha ante una efectiva realización de la educación intercultural.

Las reflexiones sobre interculturalidad y educación intercultural han sido abordadas desde los más diversos campos disciplinares: Antropología, Sociología, Filosofía, Educación, Derecho, entre otras; sin embargo, cada campo del conocimiento lo aborda desde perspectivas particulares. En este sentido, aclaramos que esta reflexión sobre la interculturalidad y las relaciones interculturales que se establecen en el interior de la escuela se hará desde una perspectiva del diálogo entre los campos del saber de la antropología y la educación. El intento es evidenciar los desafíos que necesitan ser superados en el proceso de formación intercultural. Para esto, analizamos dos realidades distintas: la política educativa intercultural para las escuelas indígenas en Brasil y la política educativa intercultural en México.

El artículo se organiza en esta introducción, en donde presentamos el tema y los objetivos, una segunda parte, donde reflexionamos teóricamente sobre los conceptos de interculturalidad y educación intercultural, para enseguida abordar las realidades concretas, es decir, de las experiencias, éstas formarán la tercera parte del artículo. Así, presentaremos la realidad brasileña y la realidad mexicana a través de un análisis de la política educativa en escuelas indígenas en Brasil a través de un relato etnográfico y un análisis de la política educativa en la educación básica en México. Finalmente, haremos algunas consideraciones finales sobre los procesos educativos interculturales, así como sobre los desafíos y posibilidades de este hacer educativo.

Interculturalidad, interculturalismo y educación intercultural: algunas concepciones desde la antropología y la educación 
En Brasil y en México, la interculturalidad constituye un campo emergente, tanto en la investigación como en la planeación de una política institucional, y más aún en una intervención pedagógica. En las diferentes vertientes académicas, políticas y educativas, el debate sobre los conceptos y soluciones interculturales refleja la influencia de tradiciones provenientes de las políticas de identidad, nacionales, regionales y étnicas (DIETZ Y MATEOS CORTÉS, 2013). En ese sentido, en los últimos años, ha surgido una amplia gama de discursos y conceptos denominados interculturales que reivindican la diferencia y el derecho a tener derechos.

Es importante aclarar que estos conceptos, emergen de los discursos sobre la diversidad cultural que han evolucionado en el tema de visualizar a la diversidad como un "problema", luego estos discursos tomaron a esta diversidad cultural como un "recurso" y posteriormente la concibieron como un "derecho". (DIETZ, 2009a , p. 330). Por lo tanto, estos discursos llevaron a la diversidad hacia el siguiente nivel conceptual, o sea la interculturalidad, el cual ha dado continuidad al proceso histórico de la lucha de las minorías étnicas. (DIETZ Y MATEOS, 2013). De esta forma, la interculturalidad considera a la diversidad cultural como un derecho y en ese sentido, debe aclararse una diferencia conceptual entre multiculturalidad e interculturalidad:

La noción de multiculturalidad, concepto descriptivo que se refiere a la coexistencia de personas y/o grupos culturalmente diferentes en espacios o territorios determinados; no se refiere a la relación entre estas personas y grupos. La interculturalidad sí se refiere a ella y la califica como una relación basada en el respeto y desde posiciones de igualdad. (SCHMELKES, 2004, p. 11).

Promover los valores universales de respeto e igualdad en el aula implica la interacción entre culturas aceptando y respetando la diferencia, sin embargo, es una labor compleja, por ello hay que señalar que la interculturalidad es:

una forma especial de relacionarse entre los individuos pertenecientes a distintas tradiciones culturales cuando conviven en el mismo territorio. Por ello se habla de interculturalidad para referirse al conjunto de objetivos y valores que deben guiar esos encuentros. Se trata, entonces, no sólo de aceptar y respetar las diferencias sino también de valorarlas positivamente y educar a los ciudadanos en los principios que guían la convivencia entre sujetos culturalmente diferentes. (PÉREZ, 2009, p. 2).

Para Tubino (2002) la interculturalidad es una respuesta al agotamiento de un proyecto moderno y la crisis del eurocentrismo. En América Latina, es una

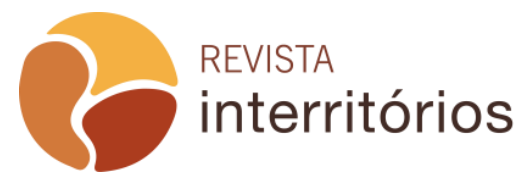

Interritórios | Revista de Educação Universidade Federal de Pernambuco, Caruaru, BRASIL | V.5 N.9 [2019] 
respuesta a la imposición cultural, a la incapacidad del estado de luchar razonablemente con los conflictos de identidades. Así, "el sentido de la interculturalidad es un intento basado en los grandes ideales de la ilustración, para manejar razonablemente el conflicto de las identidades mediante el ejercicio del diálogo y la negociación entre las partes". (TUBINO, 2002, p. 8).

La interculturalidad, según Barrio (2006) es fundada prioritariamente en el diálogo entre las diversas culturas. Puede ocurrir en los más variados contextos, ya sea a través de los medios de comunicación o incluso a través del arte, la religión y la medicina. Sin embargo, este debe ser visto como un espacio compartido de comunicación donde no hay supremacía de una cultura sobre las otras y ni siquiera una competencia, por el contrario, hay una posibilidad de colaboración.

Al reflexionar sobre esta temática, Schmelkes (2008), llama la atención en la necesidad de hacer una distinción entre interculturalidad e interculturalismo. Para ella, la interculturalidad es un concepto "descriptivo-explicativo" y se constituye como un campo de estudio de la historia, de la antropología y de la sociología, que describe y explica las relaciones entre los diferentes grupos culturales dentro de una sociedad. Como campo de estudio, la interculturalidad abarca un gran abanico de temas tales como: el racismo, la discriminación, explotación vinculada a la dominación cultural y las relaciones respetuosas entre los miembros de una sociedad. El interculturalismo, figura como un proyecto, una intervención, en el sentido de una sociedad deseada. De esta forma el interculturalismo, es:

un proyecto de nación centrado en las relaciones cualificadas entre los grupos culturales de un país: relaciones que se basan en el respeto, que se dan desde posiciones de igualdad desde el ser de cada cultura y que resultan mutuamente enriquecedoras [...] persigue el aprecio del otro diferente [...] enfatiza lo enriquecedor de las relaciones interculturales puesto que ellas abren horizontes, amplían opciones, cuestionan lo que se da por natural, favorece la criticidad, [...] es parte integrante de una democracia en una sociedad multicultural y es también ingrediente esencial de una sociedad justa y equitativa. (SCHMELKES, 2008, p. 4).

Giménez (2003) explica la distinción terminológica entre conceptos que se usan indistintamente: interculturalidad/interculturalismo (ver figura 1), en esta distinción la interculturalidad consiste en "describir o analizar las relaciones que se dan de hecho, cotidianamente, entre personas y grupos diferenciados culturalmente". (GIMÉNEZ, 2003, p. 16), mientras que el interculturalismo es "la posición o propuesta de cómo deben ser esas relaciones, en cuanto a una determinada praxis sobre el tratamiento o gestión de la diversidad cultural"

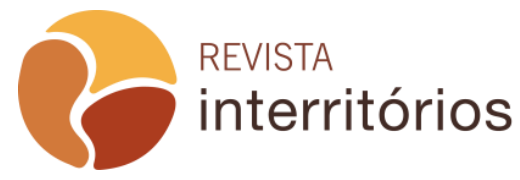

Interritórios | Revista de Educação Universidade Federal de Pernambuco, Caruaru, BRASIL | V.5 N.9 [2019] 
(GIMÉNEZ, 2003, p. 16), este interculturalismo promueve algunos ideales, lo que debería ser, bajo los principios de igualdad, de diferencia y de integración positiva (DIETZ, MATEOS, JIMÉNEZ Y MENDOZA, 2009).

Quadro 1. Pluralismo, multiculturalismo e interculturalidad. Propuesta terminológica y conceptuales.

\begin{tabular}{|c|c|c|}
\hline $\begin{array}{l}\text { Plano fáctico } \\
\text { O de los hechos } \\
\text { Lo que es }\end{array}$ & $\begin{array}{l}\text { MULTICULTURALIDAD } \\
\text { = diversidad cultural, } \\
\text { lingüística, religiosa... }\end{array}$ & $\begin{array}{c}\text { INTERCULTURALIDAD } \\
\text { = relaciones interétnicas, } \\
\text { intralingüísticas, interreligiosas... }\end{array}$ \\
\hline $\begin{array}{l}\text { Plano normativo } \\
\text { O de las } \\
\text { propuestas } \\
\text { sociopolíticas y } \\
\text { éticas } \\
\text { Lo que debería ser }\end{array}$ & $\begin{array}{l}\text { MULTICULTURALISMO } \\
\text { Reconocimiento de la } \\
\text { diferencia } \\
\text { - Principio de igualdad } \\
\text { - Principio de diferencia }\end{array}$ & $\begin{array}{l}\text { INTERCULTURALISMO } \\
\text { Convivencia en la diversidad } \\
\text { - Principio de igualdad } \\
\text { - Principio de diferencia } \\
\text { - Principio de interacción } \\
\text { positiva }\end{array}$ \\
\hline & Modalidad 1 & Modalidad 2 \\
\hline
\end{tabular}

Fonte: (GIMÉNEZ, 2003).

Lo expuesto arriba, nos muestra cómo la interculturalidad y el discurso de la educación intercultural se ha configurado desde un "triángulo atlántico" (DIETZ, 2009B), que a través de una migración del discurso aterriza en América Latina (MATEOS, 2011):

Mientras que en Estados Unidos y el Reino Unido se tiende a una educación empoderadora enfocada hacia las minorías, en la Europa continental se está optando por una educación que transversaliza el fomento de las competencias interculturales tanto de las minorías marginadas como, sobre todo, de las mayorías marginadoras. En América Latina y concretamente en México, por su parte, la educación intercultural aparece como un discurso propio en una fase postindigenista. (DIETZ Y MATEOS, 2013, p. 25).

El debate sobre interculturalidad y educación intercultural en América Latina se viene construyendo desde varias perspectivas, llegando a ser visto como antagónico. Mientras tanto, este antagonismo es muy importante para señalar que ésta es una temática en que no hay consenso y el debate no se agota, al contrario, sugiere, que el tema sea analizado desde otras perspectivas, no solamente desde una visión eurocéntrica, sino también desde una filosofía 
indígena (NASCIMENTO, 2017). En ese sentido, en el ámbito educativopedagógico y desde esta proposición, es posible analizar tanto el interculturalismo a través de los discursos institucionales y las políticas educativas, como la interculturalidad por medio de una etnografía escolar, observando sus contrastes entre los decires y los haceres (MARTíN, 1998).

Siguiendo esta perspectiva analítica y comprendiendo la interculturalidad como un diálogo entre culturas, o sea, una interrelación, como una postura de vivencia y experiencia con el otro diferente, presentamos los siguientes cuestionamientos ¿cómo se hace efectiva en la realidad concreta del aula?, ¿es posible una educación intercultural, como viene siendo defendida por un conjunto de autores?

Si asumimos la interculturalidad como un proyecto que busca el establecimiento del respeto por el otro, y aún como un proceso de reaprender a partir de la vivencia y de la experiencia nos preguntamos: ¿cómo en términos legales podríamos efectivizar ese proyecto?, ¿sería, por lo tanto, un proyecto destinado sólo a los pueblos y escuelas indígenas o a toda la sociedad?, ¿cuáles son los desafíos para la promoción y efectividad de la educación intercultural como un proyecto? Guiado por estas cuestiones vamos a analizar dos realidades concretas.

\section{Educación intercultural en Brasil}

En Brasil, desde la Constitución Federal de 1988, que aseguró a los pueblos indígenas el reconocimiento de su organización social, costumbres, lenguas y creencias, así como la garantía del derecho a la utilización de la lengua materna y los procesos propios de aprendizaje en el quehacer educativo. El Estado brasileño, viene adoptando un modelo educativo para los pueblos indígenas denominado "Específico, Diferenciado, Intercultural y Bilingüe" y tiene como objetivo el ejercicio del respeto a la diversidad étnica y cultural de cada pueblo.

De esta forma, los conceptos de diversidad e interculturalidad, que antes, eran partes de la pauta reivindicatoria de los movimientos sociales, pasaron a ser empleados por el Estado y sus agentes como fundamentos y/o principios que orientan las políticas de educación para los pueblos indígenas y las políticas públicas en general. Sin embargo, aunque sean principios orientadores de políticas públicas, los documentos oficiales no hacen una clara definición o incluso una problematización de tales conceptos (PALADINO Y ALMEIDA, 2012). 
De acuerdo con Paladino y Almeida (2012), la mayoría de los documentos oficiales referentes a Educación escolar indígena presentan una postura crítica a la concepción genérica y homogeneizante con relación a los pueblos indígenas y resalta la diversidad étnica y lingüística de los pueblos. Aun utilizan expresiones como: 'principios propios de la educación indígena, educación intercultural y pedagogía indígena', como si tales categorías fueran un consenso de la población. Un ejemplo, según las autoras, es un documento elaborado por el Ministerio de Educación - MEC- y distribuido por las secretarías estatales y municipales, escuelas indígenas, entre otras instituciones. Se trata, por lo tanto, del Referencial Curricular Nacional para las Escuelas Indígenas - RCNEI-, publicado en 1998, que tenía como principal objetivo ser el documento base para orientar el trabajo de los profesores en las escuelas indígenas.

El objetivo no es hacer un análisis del referido documento, pero es necesario hacer algunas ponderaciones, pues según Paladino y Almeida (2012: 15) el RCNEl, trae en su texto los enunciados 'interculturalidad', 'diversidad', 'pluralidad cultural' y 'multiculturalismo', sin embargo, estas categorías no son convenientemente explicadas, lo que nos incita a cuestionar ¿cuáles serían las nociones o connotaciones atribuidas a ellas? En este sentido, es imperativo comprender cómo tales conceptos vienen siendo empleados en las políticas educativas, sobre todo, el concepto de interculturalidad, pues tal concepto, es usado indistintamente por las más diversas áreas del conocimiento, y además es empleado, principalmente, para nombrar la educación escolar indígena.

El concepto de interculturalidad trae una idea de interrelación, es decir, diálogo e intercambios entre diferentes culturas y supone la coexistencia de la diversidad como riqueza. Este concepto, comenzó a ganar espacio en el campo educativo a partir de los años de 1970, cuando la diversidad étnica y cultural pasó a ser una fuente de preocupación por parte de los gobiernos dentro del movimiento social reivindicatorio hoy denominado multiculturalismo. La interculturalidad, en este sentido, propone un trato igualitario en la diversidad, sin superposiciones de una cultura sobre la otra, además, toma la diferencia como un factor de enriquecimiento y no obstáculo.

A pesar de que en los años de 1970 este concepto ganó notoriedad en el campo de la educación, Fornet-Betancourt (2004) afirma que la interculturalidad es una "disciplina pendiente", una vez que no es un debate nuevo, tampoco un llamado de ahora, ni el fruto de una difusión de una nueva moda filosófica. La interculturalidad es una demanda por justicia cultural y social, por lo tanto, una demanda histórica. De esta manera, si comprendemos la interculturalidad como una demanda por justicia, esta no puede ser vista sólo como una posición teórica o un diálogo entre culturas, pues la interculturalidad es ante todo una postura, una disposición por la cual el ser humano se capacita para acostumbrarse a vivir sus propias referencias identitarias en relación con el otro. Esta actitud, abre el 
ser humano y lo impulsa hacia un proceso de reaprendizaje, a través de la vivencia, de la experiencia, pero también, de la conciencia de que nuestras prácticas culturales deben ser también prácticas de traducción.

En el campo educativo, por ejemplo, la educación intercultural es vista como un instrumento de empoderamiento de las minorías, de las poblaciones que están a los márgenes de la cultura hegemónica. El principio que orienta ese modelo educativo está en la creencia de que las minorías, "valiéndose del dominio tanto de sus códigos específicos y de los códigos occidentales, podrían luchar por su espacio en la sociedad y en la economía mundial". (PALADINO Y ALMEIDA, 2012, p. 17).

Como vimos en la primer parte de este trabajo, no hay una comprensión única de la interculturalidad, se trata de un concepto "polifónico", pero con una convergencia en los discursos en el sentido de decir que la interculturalidad puede ser vista como un diálogo entre culturas, esto es una interacción entre los medios (FORNET-BETANCOURT, 2004); como forma de resolución de conflictos de identidad a partir del diálogo y la negociación (TUBINO, 2002); relación dialógica entre culturas (BARRIO, 2006); que tiene por objeto establecer el respeto al otro a partir del diálogo entre las culturas (SCHMELKES, 2009); y la interrelación entre culturas a través del diálogo (SORIANO, 2004). Una relación entre dos mundos, pero también, como un campo de estudio.

Así, vemos que el concepto de interculturalidad es plural, sin embargo, hay convergencia para entenderlo como un diálogo. ¿Sería, pues, esa concepción empleada en el Referencial Curricular para las Escuelas Indígenas? ¿Cómo este documento viene orientando la acción educativa intercultural?

De acuerdo con el RCNEI, las escuelas indígenas deben reconocerse como interculturales, comunitarias, específicas, diferenciadas y bilingües, pues los procesos educativos propios de las comunidades se relacionarán con la experiencia escolar, siendo que esta experiencia ha asumido varias formas a lo largo de la historia del contacto. En ese sentido, la escuela viene siendo asumida progresivamente por los pueblos indígenas, en su movimiento por autodeterminación, y viene siendo vista como:

um dos lugares onde a relação entre os conhecimentos próprios e os conhecimentos das demais culturas devem se articular, constituindo uma possibilidade de informação e divulgação para a sociedade nacional de saberes e valores importantes até então desconhecidos desta. (RCNEI, 1998, p. 24).

Según este documento, el proceso relacional permite que se proyecte, aunque como utopía, relaciones igualitarias entre los pueblos indígenas, la sociedad civil y el estado. De este modo, la escuela indígena, así como la 
educación intercultural, puede traer innumerables contribuciones a las comunidades indígenas, en el proceso de formación en busca de su autodeterminación. La escuela indígena como parte del sistema nacional de enseñanza, es un derecho a ser respetado, a través de una política pública atenta y respetuosa frente al patrimonio lingüístico, cultural e intelectual de los pueblos indígenas. Para ello, la educación escolar indígena necesita ser:

- Comunitária; porque produzida pela comunidade indígena, de acordo com seus projetos, concepções e princípios. Este princípio refere-se ao currículo, as formas administrativas, a liberdade de decisão quanto ao calendário escolar, objetivos, pedagogias espaços e momentos utilizados para a educação escolarizada.

- Intercultural; porque tem o dever de reconhecer e manter a diversidade cultural e linguística, além de promover situações de comunicação entre as experiências socioculturais, linguísticas e históricas diferentes, não considerando uma cultura superior a outra, estimular o entendimento e o respeito entre os seres humanos de identidades étnicas distintas, mesmo reconhecendo que estas relações vêm ocorrendo historicamente em contextos de desigualdade social e política.

- Bilíngue/multilíngue; porque as tradições culturais, os conhecimentos acumulado e repassado ao longo das gerações, como por exemplo: as práticas religiosas, as representações simbólicas, a organização política, projetos de futuro, são manifestados na maioria das vezes em mais de uma língua, e até mesmo os povos monolíngues em português, continuam a usar a língua de seus ancestrais como um símbolo poderoso, para onde confluem traços identitários, gerando assim um bilinguismo simbólico importante.

- $\quad$ Específica e diferenciada; porque concebida e planejada de acordo as aspirações particulares do povo, tendo autonomia em relação a determinados aspectos que regem 0 funcionamento e a orientação da educação escolar não indígena. (RCNEI, 1998, p. 24-25).

Como vemos, el RCNEI deja claro cómo debe ser la educación escolar indígena y va más allá, explica el porqué la educación destinada a los pueblos indígenas posee tales características. Así, las escuelas indígenas al asumir tales principios se constituyen como instrumentos de valorización de los saberes y procesos propios de producción y creación de la cultura, además de ser vista como un espacio de fronteras, o sea, como "espacio de interacción y contacto entre las poblaciones, donde hay flujo de personas y conocimiento y donde las diferencias sociales son construidas ". (TASSINARI, 2001, p. 64-65).

Ahora, si desde el punto de vista de la legislación está claro que la educación escolar destinada a los pueblos indígenas en Brasil es intercultural y debe atender a las especificidades arriba mencionadas, es preciso cuestionar:

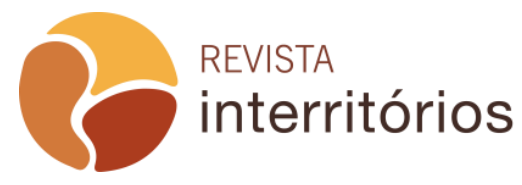

Interritórios | Revista de Educação Universidade Federal de Pernambuco, Caruaru, BRASIL | V.5 N.9 [2019] 
¿Cómo se concretiza en la práctica?, ¿cómo las comunidades indígenas vienen practicando ese modelo educativo?, ¿cuáles son los límites y los desafíos que imponen? Es, por lo tanto, sobre tales cuestiones que nos ocuparemos en el próximo ítem de este trabajo. Para ello, utilizaremos como base de nuestra reflexión la Escuela Estadual Indígena Eurico Mandulão de la Comunidad Indígena Raimundão.

\section{La educación intercultural en la escuela indígena Eurico Mandulão}

La Escuela Estadual Indígena Eurico Mandulão, está ubicada en la Comunidad Indígena Raimundão, en el interior de la Tierra Indígena del mismo nombre, en la región del Taiano, municipio de Alto Alegre, estado de Roraima. Constituida en la segunda mitad de la década de 1950, la comunidad tuvo como fundador y primer tuxaua el indio macuxi Eurico Mandulão.

La presencia de una institución escolar en la comunidad es de finales de la década de 1970, sin embargo, su reconocimiento oficial como escuela indígena y su nombre actual se dio en 2008. Con el reconocimiento oficial como escuela indígena, fue necesario realizar una adecuación en su sistema de enseñanza, conforme a las directrices nacionales que regulan el funcionamiento de las escuelas indígenas. Como escuela indígena, debería ofrecer una enseñanza basada en el respeto a la diversidad étnica y cultural allí existente, así como promover la valorización de su cultura. Este proceso de valorización cultural se daría a través de la educación intercultural, ya garantizada constitucionalmente. Es en este proceso del quehacer educativo intercultural que se presentan grandes desafíos.

De acuerdo con los profesores de la escuela Eurico Mandulão, uno de los desafíos a la implementación de la Educación intercultural, se refiere al principio comunitario en el cual si agregan otros principios. La educación escolar indígena debe ser producida en y por la propia comunidad teniendo como base sus proyectos y concepciones, garantizando a la comunidad la libertad en la construcción del currículo, formas administrativas, calendario escolar, objetivos y pedagogías.

Aunque este principio de la educación escolar indígena, establecido por el RCNEl, venga a atender los deseos de las comunidades, que, desde la década de 1970, luchan por una educación que respete sus modos de ser y hacer educación; esta autonomía parece quedar sólo en el nivel discursivo, ya que en la realidad práctica las escuelas indígenas están sometidas al mismo sistema de enseñanza de las escuelas no indígenas, no tomado en consideración sus especificidades. 
La falta de respeto al principio comunitario de las escuelas indígenas es constatada cuando reflexionamos sobre la libertad de construcción del currículo y propuesta pedagógica de la escuela. La legislación educativa, garantiza que las escuelas indígenas tengan la libertad para establecer sus propuestas pedagógicas, sus currículos, teniendo como base sus valores e intereses etnopolíticos, así como sus proyectos de sociedad y de escuela.

Sin embargo, al analizar las prácticas de las escuelas indígenas, vemos que este principio no es respetado, pues las escuelas indígenas trabajan desde una perspectiva establecida por la Secretaria del Estado de Educación, que presenta un currículo compuesto de dos partes: la nacional común obligatoria que corresponde a más o menos el $75 \%$ de los contenidos del currículo, y la diversificada que corresponde a $25 \%$ de él. La base nacional común se caracteriza por la enseñanza de las siguientes áreas del conocimiento: Lenguaje, Códigos y sus Tecnologías, Ciencias Humanas y sus Tecnologías, Ciencias de la Naturaleza, Matemáticas y sus Tecnologías; y la parte diversificada deben tener en cuenta las especificidades de la región, debiendo trabajar las cuestiones relacionadas con la diversidad cultural, pero de forma transversal.

Es, por lo tanto, esa orientación que se sigue en la Escuela Indígena Eurico Mandulão. En ella, el currículo contiene la base nacional común según lo explicitado arriba, y agrega las asignaturas de Lengua Materna y Práctica de Proyectos, sin embargo, la introducción de éstas no debe causar pérdida a la oferta de la base nacional común establecida en ley. Es válido mencionar que en lo que se refiere a la enseñanza de arte, que forma parte del área de conocimiento - Lenguajes y sus códigos, en la escuela indígena esta asignatura pasa a ser conocida como "Arte Indígena"-.

En lo que se refiere a la propuesta pedagógica de la escuela; este es más un límite que se impone a las comunidades. De acuerdo con el RCNEI, las escuelas indígenas deberán elaborar sus propuestas pedagógicas dejando claro cuáles son sus objetivos y finalidades, así como la metodología a ser adoptada en la escuela para que efectivamente la educación diferenciada ocurra. Sin embargo, al intentar elaborar sus propuestas pedagógicas las escuelas, no son respetadas. Un ejemplo, es la Escuela Estatal Indígena Eurico Mandulão, la cual llevaba más de cuatros años intentando aprobar su propuesta pedagógica de acuerdo con los anhelos de la comunidad, pero la propuesta no era aceptada por la Secretaría de Estado de Educación. Esta no aceptación de los modos de ser y hacer educación dificultaba el quehacer educativo, así como la concretización de una educación intercultural para la población en edad escolar.

La ausencia de una propuesta pedagógica, así como de un currículo propio, pone de manifiesto que la "autonomía", dada a las escuelas indígenas, queda apenas al nivel del discurso. Desde el punto de vista práctico, las escuelas indígenas son en cierto modo "obligadas" a enseñar no lo que la comunidad

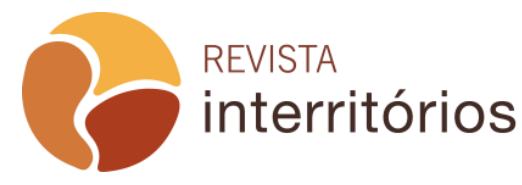

Interritórios | Revista de Educação Universidade Federal de Pernambuco, Caruaru, BRASIL | V.5 N.9 [2019] 
anhela, más bien lo que viene siendo "impuesto" por el sistema educativo nacional. Tal situación demuestra que, a pesar de que en el plano normativo se resalta el reconocimiento y la valorización de los conocimientos indígenas, en la práctica se revela una homogeneización que no considera el conocimiento indígena, por el contrario, lo jerarquiza.

Este proceso de jerarquización de los conocimientos es visible tanto en el currículo, como en las prácticas de la escuela citada. En el currículo, por ejemplo, la valorización y el reconocimiento de la cultura, así como de los conocimientos de los pueblos indígenas, se reducen a las asignaturas de lengua materna, arte indígena y práctica de proyectos. Estas con una carga horaria muy inferior a cualquiera de las otras asignaturas que componen el currículo de la escuela. En la práctica pedagógica, por ejemplo, una vez más, hay una jerarquización, ya que el contenido que viene del sistema educativo nacional es visto como conocimiento, mientras que los contenidos de las prácticas tradicionales son vistos como saberes, lo que parece dar una connotación de inferioridad. Ahora bien, si entendemos que el modelo educativo intercultural propone un trato igualitario de la diversidad, sin que haya superposición de una cultura sobre la otra, queda evidente que, desde el punto de vista normativo, el actual modelo educativo destinado a los pueblos indígenas jerarquiza más, que interculturaliza.

Al considerar la estructura curricular, percibimos que además de compartir un currículo nacional, también comparte la misma estructura organizacional de las escuelas no indígenas. A esta escuela, así como a las demás, se requiere una carga horaria de 800 horas de clase, con 200 días lectivos. Esta estructura organizativa, impuesta por las Secretarías Estatales y / o Municipales de educación, inviabiliza que las comunidades trabajen de acuerdo con sus propios sistemas.

Además de los problemas arriba mencionados, otra gran dificultad que se presenta al quehacer educativo intercultural en las escuelas indígenas es la ausencia de material didáctico específico. El libro didáctico utilizado en estas escuelas es el mismo que en las escuelas no indígenas, así como las prácticas y metodologías empleadas por la mayoría de los profesores no se diferencian en nada respecto de las escuelas no indígenas.

Otro desafío que se impone a las escuelas indígenas y ésta escuela en particular, es la falta de profesores de la propia comunidad. A esta dificultad se suma otra, la presencia de profesores no indígenas; estos últimos, no comparten la realidad cultural de la comunidad y no poseen formación específica para actuar en las comunidades indígenas.

Lo expuesto, muestra algunos de los desafíos y los límites que las comunidades indígenas enfrentan para la práctica de una educación intercultural. Eso no significa que los maestros no intenten hacerlo, éstos dicen 
que han intentado promover una educación intercultural, especifica y comunitaria, sin embargo no ha sido sencillo. Según los profesores, no es fácil dialogar con las estructuras de poder del estado que, a pesar del discurso de respeto y valorización de la diversidad, intentan imponer una propuesta homogeneizante, a través de un currículo nacional que tiende a no aceptar los conocimientos y las prácticas comunitarias como parte del currículo escolar. De esta forma, lo que se percibe es una jerarquización del saber, donde el saber tradicional construido y repasado de generación en generación por cientos de años, es visto como algo menor ante el "conocimiento científico" occidental.

En ese sentido, el gran desafío de las escuelas indígenas en la promoción de la educación intercultural es romper con estas estructuras colonialistas que aún persisten en las escuelas y comunidades indígenas. Mientras tanto, para que eso suceda, es necesario tener en cuenta que la interculturalidad va más allá de una simple relación entre culturas, que sea vista como un proceso de construcción de "otro" conocimiento, de "otra" práctica política, de "otra" forma de pensamiento en oposición a la modernidad y colonialidad como suponen Walsh (2002) y Mignolo (1999). Es necesario "descolonizar el saber", pues un pueblo no logra su autonomía a través de un acto político de separación del Estado colonizador; para que se consiga esta autonomía es fundamentalmente necesario la elaboración de "otra" ideología que sea capaz de subvertir las formas de pensar y de actuar que el estado le impone.

\section{La educación intercultural de la educación secundaria en México}

Los fenómenos sociales que acontecen en el entorno escolar, no se pueden explicar en sí mismos y desde la pedagogía, sino que se requiere de una aproximación desde el Estado-nación (DIETZ, 2003). En este sentido, la educación intercultural en México viene aparejada con planes estratégicos del Estado-nación en los cuales la educación juega un papel primordial para su consolidación, ya que es el medio por el que difundirá información bajo una metodología que esté acorde con sus ideologías e intereses.

En 2006, la educación básica, en especial, el nivel de secundaria tuvo una reforma educativa, en la cual se observa por primera vez el concepto de interculturalidad en los planes y programas de estudio (JIMÉNEZ, 2014). La Reforma de la Educación Secundaria (RES) formó parte de una nueva política educativa que se implementó al finalizar el sexenio del gobierno 2000-2006 y se concretó en el sexenio 2006-2012. Se trató de una reforma que propuso una serie de transformaciones tanto organizacionales como pedagógicas. 
En la RES se planteó el término interculturalidad como un rasgo deseable de un egresado de educación básica; el concepto fue asumido como riqueza y forma de convivencia en la diversidad social, étnica, cultural y lingüística, así como una propuesta educativa que tiene la finalidad de mejorar la comunicación y la convivencia entre distintas culturas, anteponiendo el respeto mutuo (SEP, 2006).

Así mismo, la interculturalidad fue un concepto novedoso para el Plan de Estudios de la Educación Secundaria 2006 y se incorpora en los contenidos de cada asignatura con la intención de que:

Los adolescentes comprendan que los grupos humanos forman parte de diferentes culturas, con lenguajes, costumbres, creencias y tradiciones propias. En este sentido, se pretende que los alumnos reconozcan la pluralidad como una característica de su país y del mundo, y que la escuela se convierta en un espacio donde la diversidad pueda apreciarse y valorarse como un aspecto cotidiano de la vida. (SEP, 2006, p. 19).

La interculturalidad formó parte de los contenidos transversales de dicho plan de estudios, esta interculturalidad es planteada como un discurso que expone el reconocimiento a la diferencia y promoción de la tolerancia a través de las actitudes, en el plano del ambiente y en las relaciones humanas.

De acuerdo con este escenario, la educación secundaria se reformó exaltando la singularidad del alumnado, las características específicas de los ambientes escolares y la flexibilidad didáctica:

Se propone un currículo único y nacional, que toma en consideración las distintas realidades de los alumnos; para complementarlos es necesario ser flexible en las estrategias de enseñanza y en el uso de un repertorio amplio de recursos didácticos. Toda acción de la escuela se deberá planear y llevar a la práctica a partir de un conocimiento profundo de las características particulares de sus alumnos, y considerando su interacción permanente con la sociedad a través de la familia y la escuela. (SEP, 2006, p. 15).

El discurso de la RES planteó la necesidad de que en cada escuela se genere un plan estratégico de desarrollo de acuerdo con las características propias del contexto, además de la integración de la comunidad educativa que incluye a los padres de familia, el profesorado y el estudiantado. El Plan de Estudios 2006 ofreció una libertad al profesorado para incluir en su práctica docente estrategias didácticas, temas y enfoque metodológicos acordes con las características de su ambiente escolar:

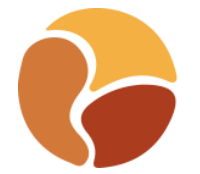


La interculturalidad es una propuesta para mejorar la comunicación y la convivencia entre comunidades con distintas culturas, siempre partiendo del respeto mutuo. Esta concepción, desde las asignaturas, se traduce en propuestas prácticas de trabajo en el aula, sugerencia de temas y enfoques metodológicos. Con ello se busca reforzar el sentido de pertenencia e identidad social y cultural de los alumnos, así como tomar en cuenta la gran diversidad social y cultural que caracteriza a nuestro país y a otras regiones del mundo. (SEP, 2006, p. 19).

Posteriormente, a finales del sexenio 2012-2018 se generó otra reforma a la educación básica, con nuevos planes y programas de estudio que va desde el nivel preescolar, primaria hasta secundaria, bajo la denominación "Aprendizajes clave para la educación integral" (SEP, 2017). Dicha reforma educativa publicada en el Diario Oficial de la Federación el 29 de junio de 2017 se organizó en tres componentes:

el primero se enfoca en la formación académica; el segundo se orienta al desarrollo personal y social de los alumnos y pone especial énfasis en sus habilidades socioemocionales; el tercer componente otorga a las escuelas un margen inédito de Autonomía curricular, con base en el cual podrán complementar el currículo -adicionales a los de los dos componentes anteriores- a las necesidades, los intereses y los contextos específicos de sus estudiantes. (SEP, 2017, p. 17).

En este momento nos preguntamos y ¿dónde quedó la interculturalidad que se había manejado en la Reforma de 2006? Entonces descubrimos que este concepto había sido suprimido y suplantado con el de habilidades socioemocionales. El término "cultura" se invisibiliza en todo el documento y se pasa a un enfoque humanista que privilegia la homogeneización del ser humano en relación con las emociones.

No obstante, esta nueva currícula lleva implementándose este ciclo escolar 2018-2019 en la educación básica y se anunció que se continuará con ella en el próximo ciclo escolar 2019-2020 y el siguiente 2020-2021, mientras se termina de diseñar la próxima reforma educativa intitulada "La nueva escuela mexicana", la cual se pretende poner en marcha en el ciclo escolar 2021-2022.

Cabe señalar que, en las elecciones de 2018, el rumbo del país tuvo un cambio de dirección notable, ya que el nuevo presidente de la República, Andrés Manuel López Obrador, trajo un proyecto de nación que, en gran medida es diferente al que se venía trabajando desde 2006. Sin embargo, nos encontramos en una pausa, donde en las aulas se continúa trabajando con los planes y

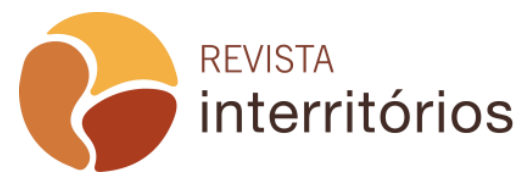

Interritórios | Revista de Educação Universidade Federal de Pernambuco, Caruaru, BRASIL | V.5 N.9 [2019] 
programas de 2011 para tercer grado, mientras que para primer y segundo grados se trabajará con los planes de 2017, mismos que en 2021 se cambiarán cuando se implemente el recién proyecto educativo de nación.

De acuerdo con un documento introductorio de la Subsecretaría de Educación Básica de la Secretaría de Educación Pública, la Nueva Escuela Mexicana (NEM) será: democrática, nacional, humanista, equitativa, integral, inclusiva, intercultural y de excelencia (SEB, 2019). Promoverá en su plan y programas de estudio la perspectiva de género, el conocimiento de matemáticas, la lectura y al escritura, la literacidad, la historia, la geografía, el civismo, la filosofía, la tecnología, la innovación, las lenguas indígenas de nuestro país, las lenguas extranjeras, la educación física, el deporte, las artes, la promoción de estilos de vida saludables, la educación sexual y reproductiva y el cuidado del medio ambiente.

Sin duda se trata de un plan de estudios ambicioso que aspira a la formación de personas técnicamente competentes y socialmente comprometidas en la solución de los grandes problemas nacionales y globales, lo que implica fortalecer la formación ciudadana. Así también, se plantea como objetivo fortalecer la formación de convicciones a favor de la justicia, la libertad y la dignidad y otros valores fundamentales derivados de los derechos humanos (SEB, 2019).

Respecto al papel que jugará la interculturalidad se menciona que a través de ésta se promoverá "la convivencia armónica entre personas y comunidades para el respeto y reconocimiento de sus diferencias y derechos, en un marco de inclusión social" (SEB, 2019). Se puede interpretar en esta primera exposición de la NEM, que la interculturalidad tendrá un lugar importante para la formación básica de los ciudadanos mexicanos, aspirando a un justicia social, ya que el plan de estudios 2017 presentó un vacío sustancial frente a los graves problemas nacionales y globales: corrupción, violencia, impunidad, pobreza, desigualdad, exclusión, discriminación, agotamiento de recursos naturales, deterioro ambiental y cambio climático.

Lo anterior nos permite reflexionar que la interculturalidad en espacios educativos constituye un concepto trascendental que debe tomarse con responsabilidad puesto que la escuela es un espacio en donde se educa y forma a los ciudadanos. Por ello, es necesario crear espacios democráticos de aprendizaje, que esta interculturalidad no se reduzca a lo axiológico o normativo, sino que se encamine hacia los haceres del profesorado y los estudiantes. Desde esta perspectiva, existe un concepto que se ha desarrollado desde el enfoque educativo por competencias, nos referimos a las competencias interculturales que, aunque no estamos de acuerdo en la totalidad de sus características, conlleva algunos elementos de nuestra propuesta, es decir, el aprendizaje 
intercultural abordado desde el término de habilidades divididas en los niveles conceptual (saber), procedimental (hacer) y actitudinal (ser).

De acuerdo con Malik (2003), las competencias interculturales constituyen los conocimientos, las habilidades o destrezas y las actitudes, que debe poseer el interlocutor / mediador intercultural, complementados por los valores que forman parte de una determinada sociedad y de los numerosos grupos sociales a los que pertenecemos. Su concepto está en estrecha relación con el modelo del enfoque educativo por competencias, pues lo abordada en tres niveles: conceptual, procedimental y actitudinal.

Malik (2003) define a cada uno de los elementos que forman parte del concepto de competencias interculturales: las actitudes interculturales, los conocimientos y las habilidades o destrezas (interpretación-comparación y aprendizaje-interacción). Aunque pone énfasis en que la base constituyente de las competencias interculturales son las actitudes.

En cuanto a las actitudes interculturales Malik (2003) expresa que "se refieren a cualidades como la curiosidad y apertura, aceptando que existen otras culturas igualmente válidas que la nuestra, la cual no es la única" (p. 437). Ello se refiere a que debe tenerse conciencia que nuestros valores no son los únicos correctos y aceptables, supone la empatía, es decir, ponerse en el lugar del otro.

En relación con los conocimientos, Malik (2003) señala que estos se refieren a lo que se conoce sobre "los grupos sociales, sus producciones y sus costumbres tanto en el propio país/zona, como en el de nuestro interlocutor, que incluye el conocimiento acerca de otras personas, de cómo se ven a sí mismas" (p. 437), y también considera los procesos generales que se dan en la interacción social, y la manera en que estos procesos influyen en todo lo anterior. Este aspecto es quizás el más laborioso del desarrollo de las competencias interculturales, pero sin duda uno de los más útiles para saber con quién nos estamos relacionando.

Malik (2003) clasifica las habilidades o destrezas en dos relaciones: interpretación/comparación y aprendizaje/interacción:

Interpretación y comparación: habilidad para interpretar, desde diversas perspectivas, hechos, ideas o documentos de otras culturas, explicarlos y relacionarlos o compararlos con la propia, para comprender cómo puede malinterpretarse fácilmente lo que alguien de otra cultura dice, escribe o hace. Aprendizaje e interacción: habilidad para adquirir nuevos conocimientos acerca de otra cultura, y la destreza de poner en práctica estos conocimientos en situaciones reales de comunicación e interacción. (MALIK, 2003, p. 437-438). 
Los componentes de la definición de competencias interculturales encierran un autoconocimiento y dominio de los valores propios, al mismo tiempo de la capacidad para entender a los demás y encontrar las líneas que se cruzan entre esas culturas. Sin embargo, Malik añade que el concepto de competencia intercultural debe ir acompañado por una conciencia cultural crítica "o habilidad para evaluar de forma crítica y con criterios explícitos las perspectivas, costumbres y producciones de la propia cultura y de las demás". (MALIK, 2003, p. 438).

En el contexto mexicano, el desarrollo de la noción de competencias interculturales es incipiente, y sólo se ha comenzado a abordar desde la educación superior (GÓMEZ, 2010; MENDOZA, 2013). Gómez (2010) desarrolló un estudio exploratorio-descriptivo sobre competencias interculturales realizado con instructores comunitarios del Consejo Nacional de Fomento Educativo (CONAFE), que brindan su servicio en la Modalidad de Atención Educativa a Población Indígena (MAEPI), en la región de San Cristóbal de las Casas, Chiapas, México. Sus resultados arrojaron que el conocimiento de la lengua indígena de la comunidad es un factor significativo que determina las competencias interculturales de los instructores y afirma que consolidar un modelo de educación intercultural implica que las instituciones educativas deben ser capaces de transformar el discurso en acción. En el caso del estudio de Mendoza (2013), realizado con estudiantes y profesores de la Universidad Veracruzana Intercultural (UVI) sede Totonacapan, obtuvo que para desarrollar las competencias interculturales en los individuos, se requieren de un mínimo de conocimientos en cuanto a las condiciones de igualdad, de justicia, de diferencia y diversidad, además de haber vivido en circunstancias de diversidad, así mismo confirmó que las competencias interculturales varían dependiendo de la situación en la que el individuo se encuentre en acción, es decir, no siempre se van a manifestar las mismas competencias interculturales. No obstante, el estudio de las competencias interculturales en México es aún incipiente y, más todavía, en la educación básica, por lo que se convierte en un desafío, ello hace necesario ahondar en la realización de otras investigaciones de estudios de casos escolares que enriquezcan el concepto y la práctica de las competencias interculturales.

En síntesis, se plantea como desafío la revalorización de la interculturalidad en la práctica docente porque constituye un enfoque que permite mejorar los procesos de enseñanza y aprendizaje a través de tomar en cuenta la diversidad cultural que conforma al estudiantado. En este sentido, es preciso que la interculturalidad, más que un rasgo del currículo, debe ser vista como un enfoque de educación intercultural a través del cual el profesorado desarrolle la práctica educativa y forme al estudiantado desarrollando habilidades para el aprendizaje intercultural. 


\section{Consideraciones finales}

En este trabajo, intentamos problematizar las cuestiones relacionadas con la diversidad cultural en el espacio educativo, llamando la atención hacia los desafíos y los límites que se imponen al quehacer educativo intercultural. Se pretende generar un debate sobre los desafíos del hacer educativo intercultural, frente a los procesos cada vez mas homogeneizantes; además de generar un diálogo entre dos campos del conocimiento: la Antropología y la Educación.

En este sentido, intentamos mostrarles cómo los conceptos de interculturalidad son percibidos y empleados, tanto en Brasil como en México y cómo los gobiernos vienen intentado promover una educación intercultural. En Brasil se piensa que la educación intercultural es para los pueblos indígenas, mientras que en México se concibe una educación intercultural para todos.

Podemos agrupar los desafíos a escala internacional, nacional y local. Desde una perspectiva internacional, vimos que tras las políticas educativas que mueven al mundo, los organismos internacionales condicionan a sus países miembros para continuar con los beneficios de préstamos, apoyos económicos, y la inclusión en sus proyectos. A escala nacional el desafío es que las políticas educativas nacionales se apropien y adapten las políticas educativas internacionales. A escala local, es que, de acuerdo con el nivel educativo que se refiera, lo adapten con sus características y sus necesidades particulares.

Entonces, el desafío se encuentra en una persona, en grupos pequeños, en los ciudadanos, que en su cotidianidad practiquen la interculturalidad, es lo que comprenden las competencias interculturales o el aprendizaje intercultural.

En la actualidad, los espacios escolares se caracterizan por ser ambientes multiculturales. Aprender a convivir para un buen desempeño en la sociedad como ciudadanos es una habilidad necesaria en la enseñanza que se lleva a cabo dentro del aula.

La interculturalidad se basa en dos principios universales: el respeto y la igualdad entre los seres humanos. Cierto que la educación formal juega un papel importante para aprender a convivir con personas que tienen una cultura distinta a la nuestra; se trata de un proceso que, de acuerdo con Artunduaga (1997), conlleva los siguientes elementos:

El respeto cultural. No se puede considerar la posibilidad de que exista interculturalidad si no se da en el marco del respeto cultural. 
- La tolerancia cultural. Se da a partir del reconocimiento del otro como parte importante en la construcción del conocimiento, valorando su propia forma de pensar y organizar su mundo.

- El diálogo cultural. A partir de un diálogo de saberes y conocimientos que permitan armonizar los principios fundamentales de cada cultura.

- El enriquecimiento mutuo. La interculturalidad implica un enriquecimiento mutuo de las culturas que entran en articulación; este enriquecimiento sólo se da si existe una apertura biunívoca de alteridad.

Promover estos elementos en el aula que implica la interacción entre culturas aceptando y respetando la diferencia, pero además logrando convivir entre ellas, es una labor compleja, por lo tanto, constituye un reto que vale la pena tomar pensando en que el ser humano cree mejores relaciones entre sus semejantes y que estas se vean reflejadas en la satisfacción personal del individuo y en el desarrollo de las sociedades.

Se entiende a la interculturalidad no solamente como una propuesta normativa, sino como una aproximación analítica al estado de las relaciones en contextos diversos (DIETZ, 2003), donde el diálogo y el conflicto están presentes.

La educación intercultural no se enfoca solamente en los grupos étnicos, sino en todos los seres humanos que coexistimos en un territorio determinado. Se trata de valorar la diversidad de los seres humanos en una interacción y tomando en cuenta las circunstancias particulares (ABDALLAH-PRETCEILLE, 2006).

En este sentido, la educación intercultural, no debe apuntar simplemente a una relación entre el conocimiento, sino que se debe traer a la mesa de debate la temática del poder que, muchas veces, es desconsiderada en este proceso. En este sentido, la propuesta de educación intercultural no debe limitarse sólo a la dimensión de la diferencia, olvidando la dimensión de la desigualdad y de las relaciones de poder y dominación a las que las minorías étnicas son sometidas, pues si no hay una preocupación con estos últimos aspectos, la educación, aunque "intercultural", continuará contribuyendo a la reproducción de las estructuras de discriminación y diferencia sociales.

Es necesario aún romper con la ideología del sistema educativo nacional, que se utiliza de la interculturalidad de forma simplista, que reduce la educación intercultural y las complejidades culturales de los pueblos indígenas a algunos símbolos como: comidas, danzas, vestido y héroes. De la misma forma, hay que romper con una postura acrítica de este mismo sistema, que coloca a la cultura como algo homogéneamente compartido por todos, sin considerar las diferencias interculturales del propio grupo.

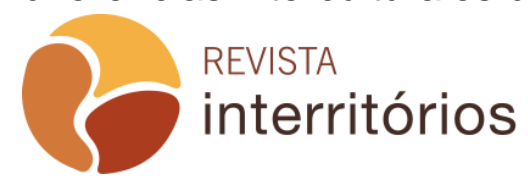

Interritórios | Revista de Educação Universidade Federal de Pernambuco, Caruaru, BRASIL | V.5 N.9 [2019] 
Por último, defendemos que la interculturalidad en el campo de la educación debe ser vista como un "proyecto", que cuestione las desigualdades que fueron construidas y jerarquizadas teniendo como base las diferencias culturales y promuevan relaciones más igualitarias cuya intención es: eliminar los muros existentes entre las diferentes culturas y establecer puentes dialógicos. En este sentido, las diferencias no deben ser vistas como sinónimo de desigualdades, sino como una pluralidad de "mundos de vidas", donde la comprensión de éstos requiere también una pluralidad de pautas comprensivas.

\section{REFERÊNCIAIS}

ABDALLAH-PRETCEILLE, Martine. Lo intercultural como paradigma para pensar la diversidad. Ponencia presentada en el Congreso Internacional de Educación intercultural. Formación del profesorado y práctica escolar. Recuperado de http://www.uned.es/congreso-inter-educacion-intercultural/pretceille_espanol.pdf, 2006.

ARTUNDUAGA, Luis Alberto. La etnoeducación: una dimensión de trabajo para la educación en comunidades indígenas de Colombia. [En línea]. Revista Iberoamericana de Educación, 13. Disponible en http://www.rieoei.org/oeivirt/rie13a02.htm, 1997.

BARRIO, Ángel B. Espina. Culturas locales iberoamericanas, comunicación e interculturalidad. In: Conocimiento local, comunicación e interculturalidad. Angel B. Espina Barrio (org) Recife: Massangana, 2006.

BRASIL, Constituição (1988). Constituição da República Federativa do Brasil: texto constitucional promulgado em 5 de outubro de 1988, com alterações adotadas pelas emendas constitucionais. Brasília: Senado Federal, subsecretaria de Edições técnicas, 2004.

DIETZ, Gunter. Multiculturalismo, interculturalidad y educación. Una aproximación antropológica. España: Universidad de Granada, 2003.

DIETZ, Gunter. El paradigma de la diversidad cultural: tesis para el debate educativo. En COMIE (ed.), IX Congreso Nacional de Investigación Educativa: Conferencias Magistrales (pp. 297-347). Mérida, Yucatán y México D.F.: Consejo Mexicano de Investigación Educativa, A.C., 2009a.

DIETZ, Gunter. Multiculturalism, Interculturality and Diversity in Education: an anthropological approach. Muenster \& Nueva York: Waxmann, 2009b.

DIETZ, Gunter. MATEOS Cortés, Laura Selene; JIMÉNEZ Naranjo, Yolanda; MENDOZA Zuany, Rosa Guadalupe. Los estudios interculturales ante la diversidad cultural, una propuesta conceptual. Revista Decisio. Septiembrediciembre, p. 26-30, 2009.

DIETZ, Gunter. MATEOS Cortés. Laura Selene. Interculturalidad y educación intercultural en México. Un análisis de los discursos nacionales e

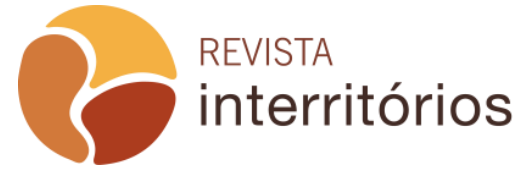


Los desafíos de la interculturalidad frente a la persistente homogeneización: una reflexión desde la realidad de Brasil y México

internacionales en su impacto en los modelos educativos mexicanos. México: SEP-CGEIB, 2013.

GIMÉNEZ Romero, Carlos. "Pluralismo, multiculturalismo e interculturalidad. Propuesta de clarificación y apuntes educativos". Educación y futuro, (8), 11-20, 2003.

GÓMEZ Nashiki, Antonio. Aplicar las competencias: una verdadera competencia. En Educación 2001, 178, marzo. México, p. 29-32, 2010.

JIMÉNEZ Lobatos, Violeta Denis. Los decires y los haceres de las competencias y la interculturalidad en la educación secundaria. Un análisis desde la Reforma de la Educación Secundaria. Tesis doctoral del Instituto de Investigaciones en Educación, UNiversidad Veracruzana, México, 2014.

MALIK, Beatriz. Intervenciones para la adquisición de competencias interculturales. En E. Repetto Talavera, Modelos de orientación e intervención psicopedagógica. Madrid: UNED, 2003.

MARTÍN Criado, Enrique. Los decires y los haceres. Papers, 56, p. 57-71, 1998.

MATEOS Cortés, Laura Selene. La migración transnacional del discurso intercultural. Su incorporación, apropiación y resignificación por actores educativos en Veracruz, México. Quito, Ecuador: Ediciones Abya-Yala, 2011.

MENDOZA Carmona, Blanca Edurne. Las competencias interculturales. Aproximaciones críticas y analíticas a la construcción de sus significados dentro de la Universidad Veracruzana Intercultural, sede Totonacapan (Tesis de maestría). Universidad Veracruzana, Xalapa, Veracruz, 2013.

NASCIMENTO, Raimundo Nonato Ferreira do. Interculturalidade e educação escolar indígena em Roraima: da normatização a prática cotidiana. Tese (Doutorado em antropologia Social) - Programa de Pós-graduação em Antropologia da Universidade Federal de Pernambuco, Recife; 2014.

NASCIMENTO, Raimundo Nonato Ferreira do. Antropologia, interculturalidade e educação escolar indígena em Roraima. 1 ed. Curitiba: Appris, 2017.

PALADINO, Mariana; ALMEIDA, Nina Paiva. Entre a diversidade e a desigualdade: uma análise das políticas públicas para educação escolar indígena no Brasil dos governos Lula. Rio de Janeiro: Contra Capa Livraria; LACED/Museu Nacional/UFRJ, 2012.

PÉREZ Ruíz, Maya Lorena. ¿De qué hablamos cuando nos referimos a lo intercultural? Reflexiones sobre su origen, contenidos, aportaciones y limitaciones. En Laura R. Valladares de la Cruz, Et. Al. (Coords.). Estados Plurales. Los retos de la diversidad y la diferencia. México: Porrúa-Juan Pablos Ed., 2009.

SCHMELKES, Sylvia. La educación intercultural: un campo en proceso de consolidación. Revista Mexicana de Investigación Educativa, 20 (9). p. 9-13, eneromarzo, 2004. Recuperado de:

http://www.comie.org.mx/v1/revista/visualizador.php?articulo=ART00399\&cri

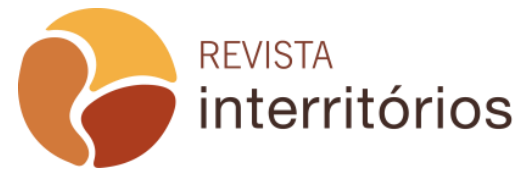


Los desafíos de la interculturalidad frente a la persistente homogeneización: una reflexión desde la realidad de Brasil y México

terio=http://www.comie.org.mx/documentos/rmie/v09/n020/pdf/rmiev09n20scB01 n01es.pdf

SCHMELKES, Sylvia. Multiculturalismo, educación intercultural y universidades. In: AGUILA, Manoel silva (org). Nuestras Unversidades y la educación intercultural. Memorias del Primer Encuentro Interuniversitario de Educación Intercultural. Manoel Silva Águila (org). Santiago do Chile, 2009. Disponível em: http://www.facso.uchile.cl/noticias/2009/educacion_superior_intercultural.htm.

SECRETARÍA DE EDUCACIÓN PÚBLICA. Educación Básica. Secundaria. Plan de estudios 2006. México: SEP., 2006.

SECRETARÍA DE EDUCACIÓN PÚBLICA. Aprendizajes clave para la educación integral. Plan y programas de estudio para la educación básica. México: SEP, 2017.

SORIANO, Ramón. Interculturalismo: entre liberalismo y comunitarismo. Almuzara, 2014.

SUBSECRETARÍA DE EDUCACIÓN BÁSICA. Modelo educativo: Nueva Escuela Mexicana. México: SEP, Mayo, 2019.

TASSINARI, Antonela Maria Inperatriz. Escola indígena: novos horizontes teóricos, novas fronteiras de educação. In: SILVA, Aracy Lopes de; FERREIRA, Mariana Kawall Leal (orgs) Antropologia, história e educação: a questão indígena na escola. 2.ed.- São Paulo: Global, 2001.

TUBINO, Fidel Arias-Schreiber. Interculturalizando el Multiculturalismo. In: Biubliotheque de la Méditerranée, Sciences humaines en Méditerranée. s/d. Disponível em:

http://ccr6.pgr.mpf.gov.br/institucional/eventos/docs_eventos/fidel_tubino.pdf.

TUBINO, Fidel Arias-Schreiber. La interculturalidade critica como proyecto Ético Político: In: encuentro continental de educadores Agostinos. Lima Peru 2005. Disponível em: http://oala.villanova.edu/congresos/educacion/lima-ponen-02.html. 